\title{
Antiaging effects of a novel facial serum containing L-ascorbic acid, proteoglycans, and proteoglycan- stimulating tripeptide: ex vivo skin explant studies and in vivo clinical studies in women
}

This article was published in the following Dove Press journal: Clinical, Cosmetic and Investigational Dermatology

\section{Aurora Garre' \\ Mridvika Narda' \\ Palmira Valderas-Martinez' \\ Jaime Piquero ${ }^{2}$ \\ Corinne Granger \\ 'Innovation and Development, ISDIN SA, Barcelona, Spain; ${ }^{2}$ Dermik Clinic, Barcelona, Spain}

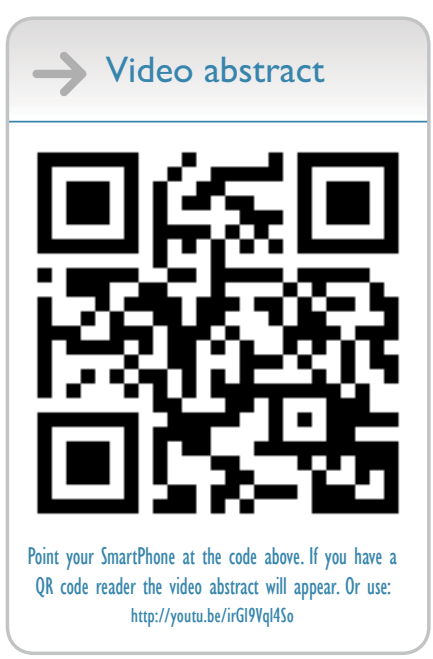

Correspondence: Aurora Garre Innovation and Development, ISDIN SA, Provençals 33, 08019 Barcelona, Spain Tel +34 932402020 ext 1210, 5563 Email aurora.garre@isdin.com
Background: With age, decreasing dermal levels of proteoglycans, collagen, and elastin lead to the appearance of aged skin. Oxidation, largely driven by environmental factors, plays a central role.

Aim: The aim of this study was to assess the antiaging efficacy of a topical serum containing L-ascorbic acid, soluble proteoglycans, low molecular weight hyaluronic acid, and a tripeptide in ex vivo and in vivo clinical studies.

Methods: Photoaging and photo-oxidative damage were induced in human skin explants by artificial solar radiation. Markers of oxidative stress - reactive oxygen species (ROS), total glutathione (GSH), and cyclobutane pyrimidine dimers (CPDs) - were measured in serum-treated explants and untreated controls. Chronological aging was simulated using hydrocortisone. In both ex vivo studies, collagen, elastin, and proteoglycans were determined as measures of dermal matrix degradation. In women aged 21-67 years, hydration was measured up to 24 hours after a single application of serum, using Corneometer and hygrometer. Subjects' perceptions of efficacy and acceptability were assessed via questionnaire after once-daily serum application for 4 weeks. Studies were performed under the supervision of a dermatologist.

Results: In the photoaging study, irradiation induced changes in ROS, CPD, GSH, collagen, and elastin levels; these changes were reversed by topical serum application. The serum also protected against hydrocortisone-induced reduction in collagen, elastin, and proteoglycan levels, which were significantly higher in the serum-treated group vs untreated hydrocortisonecontrol explants. In clinical studies, serum application significantly increased skin moisture for 6 hours. Healthy volunteers perceived the product as efficient in making the skin brighter, more hydrated, and decreasing wrinkles and wished to continue using it. The serum was well tolerated and noncomedogenic.

Conclusion: The serum protected against oxidative damage and dermal protein loss caused by photo- and chronological aging in human skin explants. In-vivo, the serum hydrated skin for 6 hours, and users perceived increased skin brightness, hydration, and fewer wrinkles.

Keywords: antiaging, antioxidant, proteoglycans, L-ascorbic acid, vitamin C, tripeptide

\section{Introduction}

Visible signs of skin aging include altered skin texture, dryness, loss of firmness, and wrinkles. These are undesirable, and consumers demand effective products with immediate and long-term effects. While there is no shortage of cosmetic antiaging products on the market, there is great variation in their efficacy and the potency of their ingredients. Ampoules of serum are attractive to many consumers because they 
are often associated with higher concentrations of ingredients and are perceived as higher-quality luxury products.

The clinical signs of aging develop due to multiple factors, but loss of dermal proteins is central: decreased collagen and elastin levels result in a disorganized extracellular matrix (ECM) manifesting clinically as wrinkles and loss of firmness. ${ }^{1}$ Loss of proteoglycans, normally responsible for binding matrix protein fibrils and hyaluronic acid - which has a high capacity for binding water - leads to altered, drier skin texture. ${ }^{2}$ Oxidation also plays a key role and has been described as the most important factor in skin aging. ${ }^{3}$ Oxidative stress can be induced by many factors, but the final common pathway involves the formation of reactive oxygen species (ROS) and downstream events including the formation of cyclobutane pyrimidine dimers (CPDs), the degradation of dermal ECM components, and the reduction of skin antioxidant reserves. ${ }^{3,4}$

In response to consumer demand for highly effective antiaging products, and based on the established physiology of skin aging, we developed a new cosmetic product to provide immediate and long-term antiaging effects. Proteoglycans and low molecular weight hyaluronic acid were included to provide immediate "flash-effect" hydration; for longerterm effects, we combined the antioxidants L-ascorbic acid and ergothioneine with a proteoglycan-stimulating peptide (tetradecyl aminobutyroylvalylaminobutyric urea trifluoroacetate) and fragmented proteoglycans. The combination of this peptide with proteoglycans has been commercially termed Ultraglycans ${ }^{\mathrm{TM}}$.

We tested the product in a total of five ex vivo and in vivo studies to assess its efficacy against factors involved in aging, including oxidation and dermal matrix damage.

\section{Materials and methods}

\section{Ex vivo studies: photoaging and photo- oxidation and chronological aging}

\section{Sample acquisition for ex vivo studies}

Abdominal skin samples were obtained with consent from healthy 40- to 55-year-old women undergoing plastic surgery, in accordance with French Law LOI no 2011-814 of July 7, 2011 , on bioethics. ${ }^{5}$ Within 2 hours after surgery, skin was dermatomed to remove excess fat and tissues, cut into $0.8 \mathrm{~cm}^{2}$ pieces, and placed with epidermis facing up in culture plates containing Dulbecco's Modified Eagle's Medium (DMEM; Sigma-Aldrich Co., St Louis, MO, USA) with 1\% penicillinstreptomycin. Cultures were incubated for 48 hours at $37^{\circ} \mathrm{C}$ with $5 \% \mathrm{CO}_{2}$ for recovery prior to study initiation.

\section{Induction of skin aging}

Photoaging and photo-oxidation studies

\section{Photoaging study}

To simulate photoaging, human skin explants in culture were irradiated at $10 \mathrm{~J} / \mathrm{cm}^{2}$ of UV/visible/IR light using the SOL 500 solar simulator (Honle UV America Inc., Marlborough, MA, USA) for 7 days in a 10-day period (not irradiated over the weekend). Facial serum was applied topically daily to skin explants after each irradiation $\left(2 \mathrm{mg} / \mathrm{cm}^{2}\right)$. Nonirradiated control (NIC) and irradiated control (IC) groups were not treated with serum but maintained in the same conditions as the intervention group (three explants/group). On day 10, 24 hours after the last irradiation and product application, tissues were removed from culture and processed for collagen and elastin quantification. One explant per group was processed and embedded in paraffin for histological analysis.

\section{Photo-oxidation study}

To study immediate oxidative stress due to UV exposure, skin explants were irradiated once only (same conditions of radiation as described earlier). The facial serum was applied 24 hours after irradiation and left on overnight. The following three groups were compared: irradiated and serum treated (IST), IC, and NIC. Nonirradiated and ICs were not treated with serum but maintained in the same conditions as the intervention group (IST) (three explants/group). The tissues were processed to determine levels of ROS, CPDs, and total glutathione (GSH).

\section{Chronological skin aging simulation: hydrocortisone- induced skin aging}

Human skin explants were distressed with continuous exposure to hydrocortisone ( $10 \mu \mathrm{g} / \mathrm{mL}$ added each day) in culture media for a total of eight applications to induce skin atrophy and simulate chronological aging. Previous studies have found that glucocorticoids decrease collagen and skin thickness. ${ }^{6}$ Serum was applied topically daily $\left(2 \mathrm{mg} / \mathrm{cm}^{2}\right)$ at the same time as hydrocortisone was added. Control group explants received hydrocortisone only in culture media. At day 10, all tissues were removed from culture media and processed for the quantification of elastin, soluble collagen, proteoglycans, and hyaluronic acid. One explant per group was processed and embedded in paraffin for histological analysis.

\section{Product application, viability studies}

In both ex vivo studies, facial serum was applied topically using a micropipette, at $2 \mathrm{mg} / \mathrm{cm}^{2}(\sim 10 \mu \mathrm{L})$ per application, 
and spread using a microspatula to ensure homogenous application. Viability and metabolic activity analysis were performed with lactate dehydrogenase (LDH) and resazurin assays. For the LDH assay, an aliquot of culture media removed from each skin sample was reacted with formazan dye and absorbance was read at appropriate wavelength. ${ }^{7}$ For the resazurin assay, an aliquot of media surrounding the skin explants was removed prior to irradiation or hydrocortisone treatment, and treated with resazurin in $\mathrm{NaCl}$ solution for 1 hour. Subsequently, resazurin was quantified using a fluorometer plate reader. ${ }^{8}$

\section{Collagen, elastin, and proteoglycan quantification}

Collagen content was quantified using a dye-binding method (Sircol $^{\mathrm{TM}}$ Collagen Assay; Biocolor Ltd, Carrickfergus, UK), and elastin was quantified using a Fastin ${ }^{\mathrm{TM}}$ Elastin kit (Biocolor Ltd.), in both cases following the manufacturer's instructions. Proteoglycans were quantified using the Glycosaminoglycan Assay Blyscan ${ }^{\mathrm{TM}}$ kit (Biocolor Ltd.), following the manufacturer's instructions.

\section{Quantification of ROS, GSH, and CPDs}

For the determination of ROS, CPDs, and GSH, a suspension of isolated human skin cells was prepared. For this, skin tissue was minced and digested in $3.5 \mathrm{mg} / \mathrm{mL}$ collagenase in DMEM for 4 hours at $37^{\circ} \mathrm{C}$ and filtered. The cellular filtrate was then enriched for dermal cells by centrifugation (1700 rpm, $5 \mathrm{~min}$ ), and the resultant pellet was resuspended in DMEM.

Total GSH concentration was determined with an enzymatic recycling reaction using a commercially available kit (OxiSelect ${ }^{\text {TM }}$ Total Glutathione [GSSG/GSH] Assay Kit; Cell Biolabs, Inc., San Diego, CA, USA) according to the manufacturer's instructions. All reagents and the GSH standard curve were prepared immediately before use.

Intracellular ROS levels were determined with the oxidant-sensitive fluorescent probe $2^{\prime}, 7^{\prime}$-difluorescein diacetate (DFFH-DA) and flow cytometry. Briefly, human skin cell suspensions were washed with cold phosphate-buffered saline (PBS), centrifuged, and resuspended. Subsequently, the cells were loaded with DFFH-DA, at a final concentration of $10 \mu \mathrm{M}$, and incubated for $30-40$ minutes at $37^{\circ} \mathrm{C}, 5 \% \mathrm{CO}_{2}$. Afterward, the cells were washed with PBS and cell fluorescence was assessed at $4^{\circ} \mathrm{C}$ by flow cytometry (Cytomics FC 500 MCL; Beckman Coulter, Inc., Brea, CA, USA).

CPDs were determined by enzyme immunoassay using anti-CPD antibody in a commercially available kit, Oxiselect $^{\mathrm{TM}}$ UV-Induced DNA Damage ELISA (STA-322;
Cell Biolabs, Inc.). DNA was extracted from dermal tissues using an extraction kit (Dynabeads DNA DIRECT Universal Kit; Thermo Fisher Scientific, Waltham, MA, USA) and converted into single-stranded DNA by incubating the sample at $95^{\circ} \mathrm{C}$ for 10 minutes and rapidly chilling on ice for 10 minutes. CPDs were quantified by binding with an anti-CPD antibody followed by an HRP-conjugated secondary antibody. The CPD level was determined by comparing against a standard curve prepared from predetermined CPD-DNA standards.

\section{Tissue collection, processing, and staining}

Masson's trichrome stain (Goldner variant) was used in both ex vivo studies to detect collagen and general tissue morphology.

Skin explants were fixed in $10 \%$ buffered formalin for 48 hours and processed overnight in increasing concentrations of ethanol, followed by three washes with xylene and subsequent equilibration in paraffin. Tissues were then embedded in paraffin blocks and cut into $5 \mu \mathrm{m}$ sections. For staining, sections were deparaffinized, washed with xylene, and rehydrated for the preparation of Masson-Goldner or Alcian blue staining. For Masson-Goldner, the MassonGoldner staining kit (1.00485; Sigma-Aldrich Co.) was used as per the manufacturer's instructions. In the hydrocortisone study, Alcian blue was used to stain proteoglycans. For this, samples were incubated with Alcian blue (8GX, A3157; Sigma-Aldrich Co.) for 30 minutes and rinsed with deionized water. Finally, slides were washed with $1 \%$ acetic acid and xylene and mounted.

\section{Statistical analysis for ex vivo studies}

Homogeneity of variance was confirmed by Levene's test, and normality was confirmed by the Anderson-Darling test. One-way analysis of variance (ANOVA) with BonferroniDunn's correction was used to check for overall differences in variance, and an unpaired $t$-test was used to assess differences between serum-treated samples against control groups. $P$-values $<0.05$ were considered statistically significant.

\section{Clinical studies: hydration kinetics, subject self-evaluation under dermatological control, and consumer test}

Clinical studies were conducted according to the guideline for Good Clinical Practice and the Declaration of Helsinki. ${ }^{9}$ Due to being cosmetic product studies, ethics committee approval was not required. All subjects gave written informed consent. 


\section{Hydration kinetics study}

\section{Subjects}

Between March and April 2015, a single-center open randomized study was conducted to assess skin hydration levels after a single application of the investigational product in 20 female subjects. Exclusion criteria were as follows: taking part in another study, pregnancy (or planned) or breastfeeding during the study, and medical history of a dermatological condition liable to interfere with study data, as assessed on taking a medical history.

\section{Protocol}

Before baseline measurements, subjects were instructed not to apply any moisturizing products for 72 hours and to wash their forearms with water on the morning of the visit to the investigational unit. In each subject, two $4 \times 4 \mathrm{~cm}$ squares were marked on each volar forearm. Each square was divided into four $1 \mathrm{~cm}$ wide zones for measurements at 1 hour $(\mathrm{T} 1 \mathrm{H})$, 3 hours (T3H), 6 hours (T6H), and 24 hours (T24H). Before measuring hydration, subjects spent at least 20 minutes in a temperature- and humidity-controlled environment, where they remained until after the $\mathrm{T} 6 \mathrm{H}$ reading. Once baseline measurements were taken, $2 \mathrm{mg} / \mathrm{cm}^{2}$ of the product was applied with a finger stall by a technician to two of the four squares, according to a randomization protocol, and the other two squares served as untreated controls. Four squares were used to allow measurements to be repeated. Skin moisture was assessed by measuring electrical capacitance with a corneometer (MPA 580; Courage + Khazaka Electronic $\mathrm{GmbH}$, Cologne, Germany) as well as via an impedance method with a skin surface hygrometer (Skicon-200EX; IBS Co., Ltd., Hamamatsu, Japan). After the T6H measurement, subjects went home with instructions to keep the area dry and not apply any other products before the T24H measurement. During the study, they were not allowed to consume substances likely to induce sweating (eg, coffee).

\section{Data analysis}

Treated zones were compared with untreated control zones at each time and for both measurement techniques. A RyanJoiner test was performed at $\mathrm{T} 0 \mathrm{H}$ to assess normality for differences between the treated area and the untreated control area. If the differences followed a normal distribution, a Student's $t$-test was used on paired data; if not, a Mann-Whitney test was used. For each time (Tx) thereafter, the differences $\mathrm{Tx}-\mathrm{T} 0$ were calculated for the investigational product $(i)$ and the control (c): $(\mathrm{Tx}-\mathrm{T} 0)(i)$ - [Tx-T0](c). A $P$-value of $<0.05$ was considered statistically significant.

\section{Subject-perceived acceptability and efficacy study with dermatological appraisal of cutaneous acceptability and comedogenesis}

A single-center open study under dermatological control was carried out to assess acceptability in 30 subjects (mean age 37 years), in March-April 2015, in Barcelona, Spain. Subjects were otherwise healthy, as assessed on taking a medical history, with a maximum $25 \%$ of participants with a history of atopy, reflective of the incidence in the general Spanish population. Subjects were instructed to apply the serum to the face once daily, representing conditions of normal use, for 4 weeks, and use their usual cleansing products concomitantly. On days 1 and 29, a dermatologist assessed each subject for macroscopically visible signs of cutaneous irritation and comedones. On day 29, subjects completed evaluation questionnaires on efficacy and acceptability (closed questions) at home. Cutaneous acceptability was assessed using a modified questionnaire and included any reactions noticed by the subjects or the dermatologist, reactions requiring modification or interruption of application of the product, reactions considered as "pertinent", and any adverse reactions. For statistical analysis of comedogenesis, a nonparametric two-tailed Wilcoxon test was used. $P<0.05$ was considered statistically significant.

\section{Consumer test}

A single-center open study was conducted to assess the acceptability and efficacy of the investigational product after 4 weeks of use in 105 women (mean age 35.7 years). As with the above study, participants were healthy with a maximum $25 \%$ with a history of atopy. Participants applied the serum at home on alternate days and used their usual cosmetic products concomitantly. At 4 weeks, participants completed a questionnaire on the product's characteristics and effects. The questionnaire included open questions, closed questions, a 4-point Likert scale for efficacy and cosmetic characteristics, and yes/no questions on cutaneous and ocular reactions to assess acceptability. Participants also rated the product overall on a scale of 1-10.

\section{Results}

\section{Ex vivo photoaging and photo-oxidation studies Photoaging study \\ Viability}

Solar radiation led to a significant increase in LDH levels in the IC group compared to the NIC group. In the IST group, the product interfered with the LDH assay, so the results 
could not be used. Resazurin levels in the IC group decreased significantly (64.58\% at day 10 ) compared to the NIC group $(100 \%)$, indicating a significant reduction in skin metabolic activity. The IST group had higher resazurin values (163.05\% at day 10) than the IC group and, in fact, was higher than the NIC group from day 1 onward (Figure 1).

\section{Dermal matrix parameters}

Collagen and elastin levels were significantly reduced in the IC group compared to the NIC group after 9 days: a $40.39 \%$ reduction in collagen and $20.60 \%$ reduction in elastin levels (Table 1). This change was evident in histological sections stained with Masson-Goldner's stain where greater alteration of collagen fibers and fibrin degradation (muscle) was observed in irradiated tissues compared to NIC tissues.

In the IST group, collagen levels were higher without reaching statistical significance $(P=0.092)$ and elastin levels were significantly higher $(P<0.05)$ than in the IC group

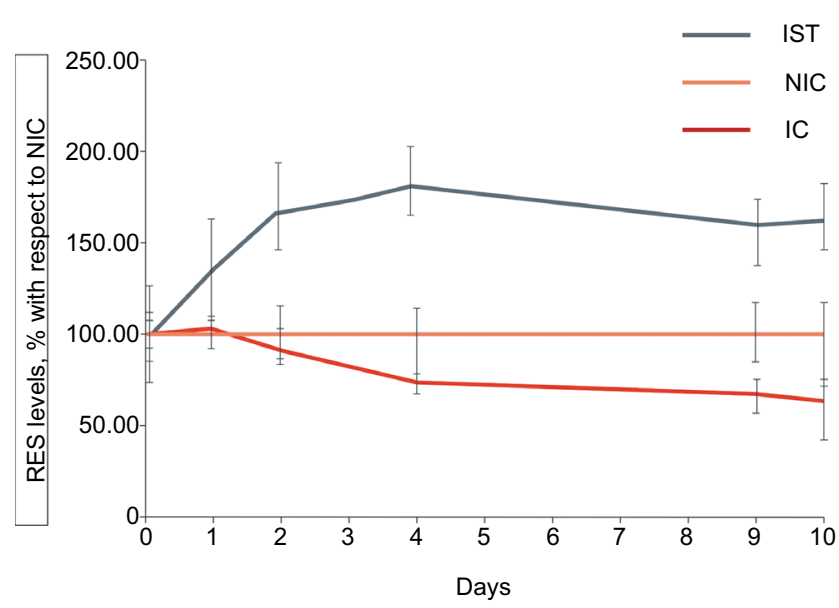

Figure I Resazurin levels, indicating cell viability, in ex vivo photoaging and photooxidation study.

Notes: Expressed as a percentage compared to NIC levels (control considered $100 \%$, mean \pm standard deviation in NIC, IC, and IST.

Abbreviations: IC, irradiated control; IST, irradiated and serum treated; NIC, nonirradiated control; RES, resazurin.
(Figure 2). Table 2 shows the $P$-values from statistical analysis between the different groups. On histological sections stained with Masson-Goldner stain, the dermis of the serum-treated group was well preserved and resembled that of nonirradiated skin explants (Figure 3).

\section{Photo-oxidation study}

The single exposure to solar radiation led to a significant increase in ROS-positive cells and CPDs and a reduction in total GSH levels compared to NICs. The percentage of ROS-positive cells was significantly lower in the IST group than in the IC group and was similar to levels in the NIC group. CPD levels were also significantly lower in the IST group than in the IC group $(P<0.05)$ and were similar to the NIC group (Figure 4). GSH levels in the IST group were significantly higher than in the IC group. Table 3 shows the $P$-values from statistical analysis between the different groups.

\section{Hydrocortisone aging study \\ Viability}

LDH assay results could not be used as the product interfered with assay. There was no significant reduction in resazurin in the hydrocortisone control (HC) group compared to the non-

Table $2 P$-values from statistical analysis in photoaging and photo-oxidation study: unpaired t-test comparisons between experimental groups

\begin{tabular}{lll}
\hline Groups compared & $\boldsymbol{P}$-values \\
\cline { 2 - 3 } & Collagen & Elastin \\
\hline IC vs NIC & $0.027^{*}$ & $0.002^{*}$ \\
IC vs IST & 0.092 & $0.006^{*}$ \\
NIC vs IST & 0.253 & 0.554 \\
\hline
\end{tabular}

Note: *Statistically significant.

Abbreviations: IC, irradiated control; IST, irradiated and serum treated; NIC, nonirradiated control.

Table I Ex vivo study, antioxidant, and antiphotoaging parameters

\begin{tabular}{llll}
\hline Parameter & IST & IC & NIC \\
\hline Antioxidant parameters & & $32.58(4.34)$ & $14.68(4.04)$ \\
ROS, \% of positive cells & $16.44(3.30)$ & $79.67(2.39)$ & $113.61(5.32)$ \\
Glutathione, nmol/L & $97.94(2.56)$ & $3.30(0.75)$ & $0.8 \mathrm{I}(0.5 \mathrm{I})$ \\
CPD, $\mathrm{ng} / \mathrm{mL}$ & $1.23(0.32)$ & & \\
ECM parameters & & $1.20(0.53)$ & $2.01(0.54)$ \\
Collagen, $\mu \mathrm{gg} / \mathrm{mg}$ & $\mathrm{I} .69(0.34)$ & $59.61(26.19)$ & $100(2.01)$ \\
Collagen, \% level with respect to healthy group & $83.97(16.87)$ & $6.79(0.84)$ & $8.56(0.83)$ \\
Elastin, $\mu \mathrm{g} / \mathrm{mg}$ & $8.45(0.90)$ & $79.40(9.79)$ & $100(7.5 \mathrm{I})$ \\
Elastin, \% level with respect to healthy group & $98.84(10.54)$ & & \\
\hline
\end{tabular}

Note: Mean \pm standard deviation (SD).

Abbreviations: CPD, cyclobutane pyrimidine dimer; ECM, extracellular matrix; IC, irradiated control; IST, irradiated and serum treated; NIC, nonirradiated control; ROS, reactive oxygen species. 

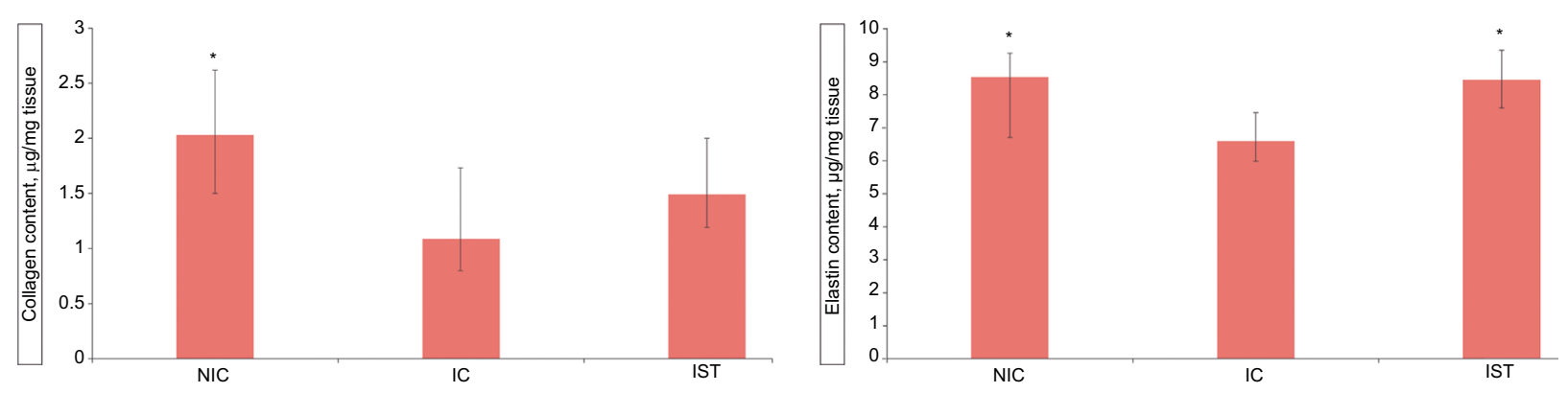

Figure 2 Antiphotoaging study, mean collagen, and elastin levels at the end of the study (day 10 ).

Note: ${ }^{*} P<0.05$ compared with IC.

Abbreviations: IC, irradiated control; IST, irradiated and serum treated; NIC, nonirradiated control.

A

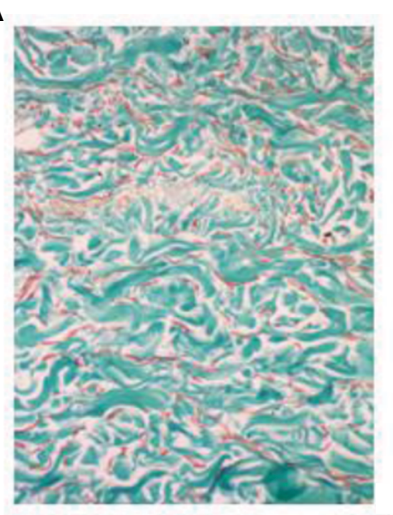

B

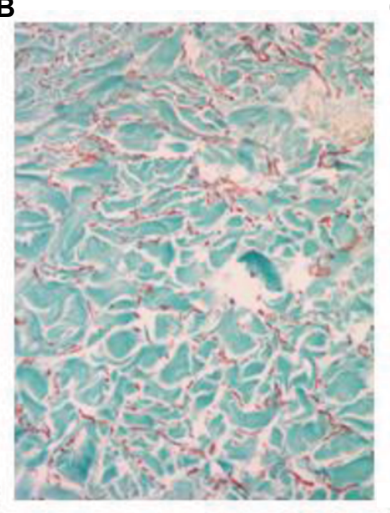

C

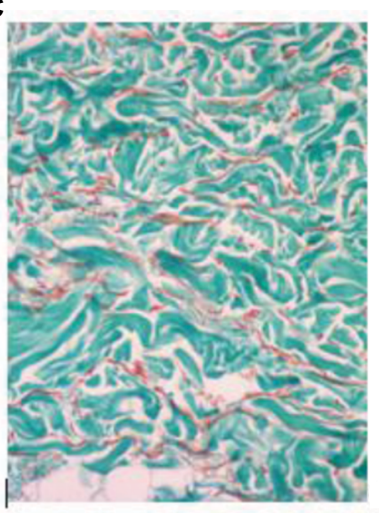

Figure 3 Masson-Goldner staining of dermal tissue in the ex vivo photoaging study, with connective fibers stained blue-green, fibroblast nuclei stained dark brown-black, and fibroblast cytoplasm stained light red-pink (magnification 200x).

Notes: (A) Untreated control tissue; (B) irradiated control tissue; and (C) irradiated and serum-treated tissue.

A

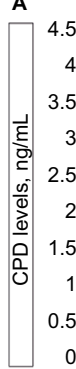

4.5
4.
3.5
3
2.5
2
1.5
1
0.5
0

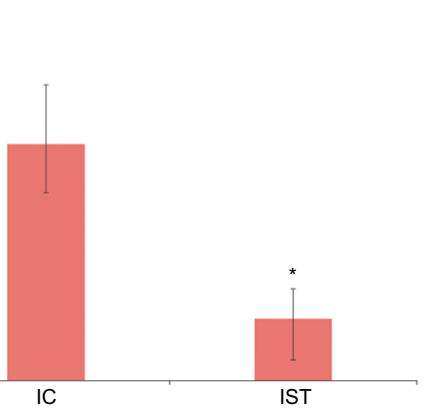

C
B

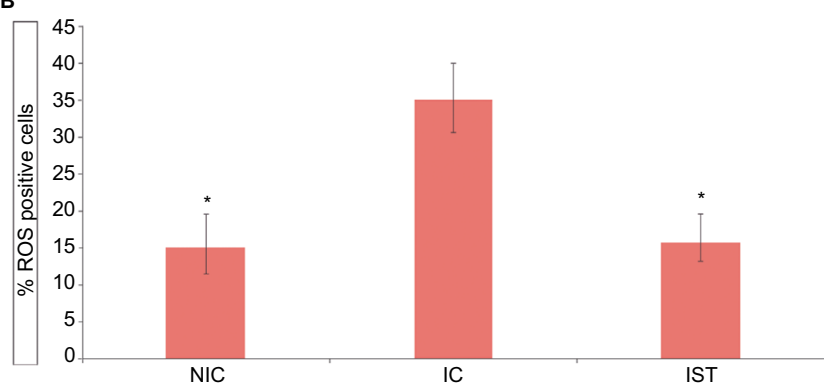

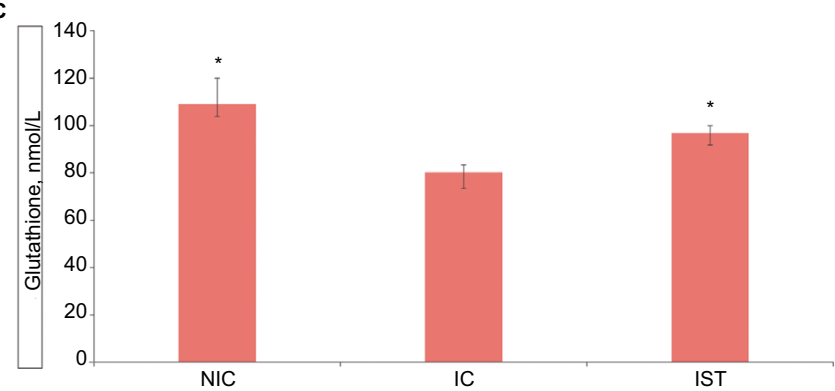

Figure 4 Markers of oxidation in the antioxidant and antiphotoaging study.

Notes: (A) CPDs; (B) percentage of ROS-positive cells; and (C) total glutathione levels in NIC, IC, and IST. $* P<0.05$ compared with IC.

Abbreviations: CPDs, cyclobutane pyrimidine dimers; IC, irradiated control; IST, irradiated and serum treated; NIC, nonirradiated control; ROS, reactive oxygen species. 
HC (NHC) group. In the hydrocortisone- and serum-treated (HST) group, resazurin values increased beyond the NHC values (156.21\%, with 100\% representing the NHC) (Figure 5).

\section{Collagen and elastin}

Skin explants exposed to hydrocortisone in culture medium over 10 days exhibited significantly reduced levels of collagen (21.39\% reduction) and elastin (27.24\% reduction) compared

Table 3 P-values from statistical analysis in photoaging and photo-oxidation study: unpaired $t$-test comparisons between experimental groups

\begin{tabular}{llll}
\hline $\begin{array}{l}\text { Groups } \\
\text { compared }\end{array}$ & \multicolumn{2}{l}{$\boldsymbol{P}$-values } & \\
\cline { 2 - 4 } & CPDs & ROS & Glutathione \\
\hline IC vs NIC & $0.002^{*}$ & $<0.00 I^{*}$ & $<0.00 I^{*}$ \\
IC vs IST & $0.00 I^{*}$ & $<0.00 I^{*}$ & $<0.00 I^{*}$ \\
NIC vs IST & 0.130 & 0.417 & $<0.00 I^{*}$ \\
\hline
\end{tabular}

Note: *Statistically significant.

Abbreviations: CPDs, cyclobutane pyrimidine dimers; IC, irradiated control; IST, irradiated and serum treated; NIC, nonirradiated control; ROS, reactive oxygen species.

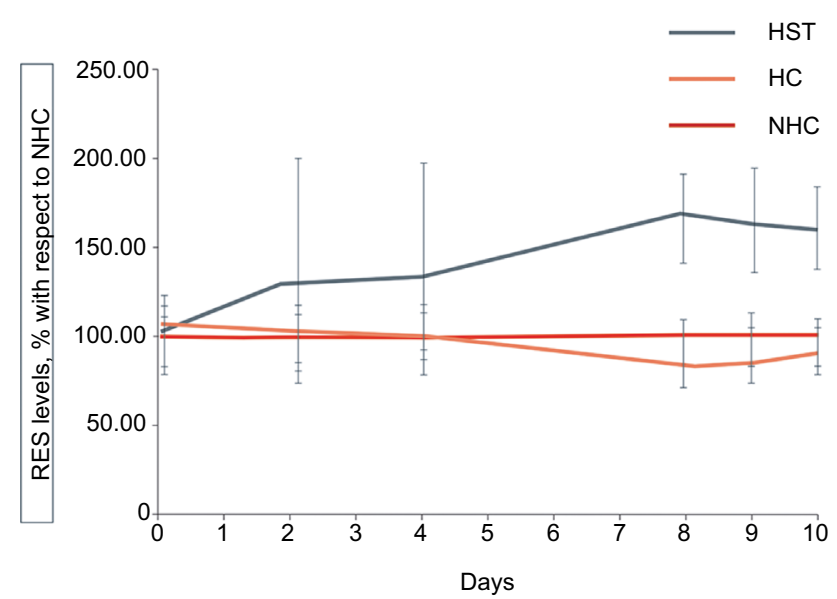

Figure 5 Resazurin levels, indicating cell viability, throughout the hydrocortisone aging study.

Note: Expressed as a percentage compared to nonaged control levels (control considered $100 \%$ ), mean \pm standard deviation.

Abbreviations: HC, hydrocortisone control; HST, hydrocortisone and serum treated; NHC, non-HC; RES, resazurin. with NHC explants, indicating that dermal morphology resembling aged skin had been produced. In HST explants, collagen content was significantly higher $(P<0.05)$ than in the $\mathrm{HC}$ group and similar to the NHC group. Elastin values in the HST group were also similar to those in the NHC group (Table 4). The changes in collagen fiber arrangement and fibroblast structure in the untreated control, hydrocortisonetreated, and HST sections can be seen in the Masson-Goldner stained sections (Figure 6).

\section{Proteoglycans}

A $21.24 \%$ reduction in proteoglycan content was observed in the HC group compared to the NHC group $(P<0.05)$. The HST group had significantly more $(P<0.05)$ proteoglycan content compared with the HC group (92.56 \pm 7.70 vs $78.76 \pm 11.61$, respectively). The HST group had values similar to the NHC group (no statistical differences, $92.56 \pm 7.70$ vs $100 \pm 8.79 \%$, respectively). Histological analysis of Alcian blue-stained sections of the HC group showed a loss of proteoglycans, seen as a deep blue color, unlike the HST skin, which showed an adequate density of proteoglycans (Figure 7).

The results suggest that topical application of facial serum helped preserve levels of collagen, elastin, and proteoglycans in the skin despite the presence of hydrocortisone in culture media, which otherwise led to clear signs of aging in dermal ECM components.

\section{Clinical studies: hydration kinetics, subject self-evaluation under dermatological control, and consumer test}

The characteristics of the clinical studies subjects are shown in Table 5.

\section{Hydration kinetics study}

Skin moisture on corneometer reading (arbitrary units [AU]) increased significantly from a mean (SD) of 36.10 (6.19) at baseline to $51.97(11.55)$ at $1 \mathrm{~h}, 43.16(6.12)$ at $3 \mathrm{~h}$, and 40.9

Table 4 Outcome variables for antiaging efficacy in the hydrocortisone ex vivo study

\begin{tabular}{|c|c|c|c|}
\hline Antiaging parameters & HST & HC & NHC \\
\hline Collagen, $\mu \mathrm{g} / \mathrm{mg}$ & $3.34(0.58)$ & $2.56(0.4 I)$ & $3.25(0.48)$ \\
\hline Collagen, \% level with respect to healthy group & $102.63(17.94)$ & $78.61(12.63)$ & $100.00(14.86)$ \\
\hline Elastin, $\mu \mathrm{g} / \mathrm{mg}$ & $10.7 \mid(0.66)$ & $8.41(0.39)$ & I I.04 (I.46) \\
\hline Elastin, \% level with respect to healthy group & $93.72(5.19)$ & $72.76(3.39)$ & $100.00(11.11)$ \\
\hline Proteoglycan & $0.76(0.06)$ & $0.64(0.09)$ & $0.82(0.07)$ \\
\hline Proteoglycan, \% with respect to healthy group & $92.56(7.70)$ & $78.76(11.61)$ & $100.00(8.79)$ \\
\hline Hyaluronic acid, $\mu g / m g$ skin & $0.04(0.03)^{* *}$ & $0.18(0.04)$ & $0.27(0.06)$ \\
\hline Hyaluronic acid, \% with respect to healthy group & $14.72(9.96)^{* *}$ & $64.83(13.90)$ & $100(23.82)$ \\
\hline
\end{tabular}

Note: **In the hyaluronic assay, the study product interfered with the colorant, so results were inconclusive in the serum-treatment group. Data are presented as mean (SD). Abbreviations: HC, hydrocortisone control; HST, hydrocortisone and serum treated; NHC, nonhydrocortisone control. 
A

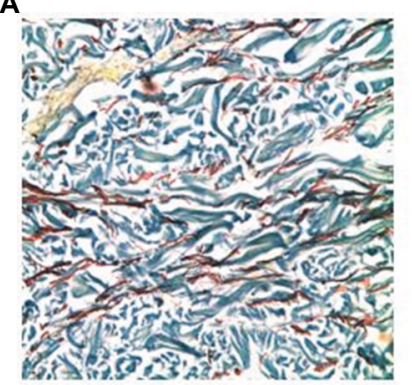

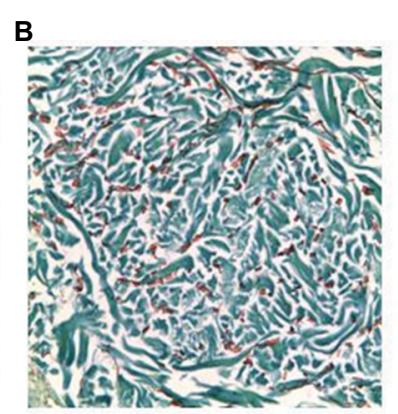

C

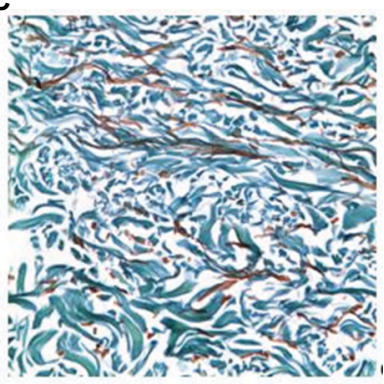

Figure 6 Masson-Goldner staining of dermal tissue in the ex vivo hydrocortisone study, with connective fibers stained blue-green, fibroblast nuclei stained dark brown-black, and fibroblast cytoplasm stained light red-pink (magnification $200 \times$ ).

Notes: (A) Untreated control tissue; (B) hydrocortisone control tissue; and (C) hydrocortisone- and serum-treated tissue.

A

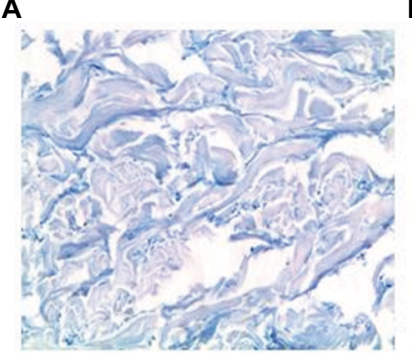

B

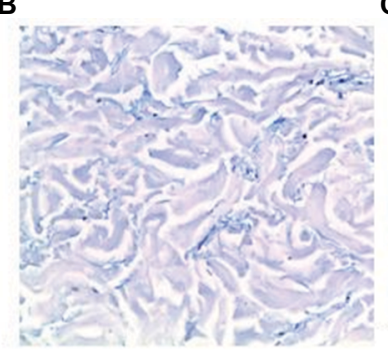

C

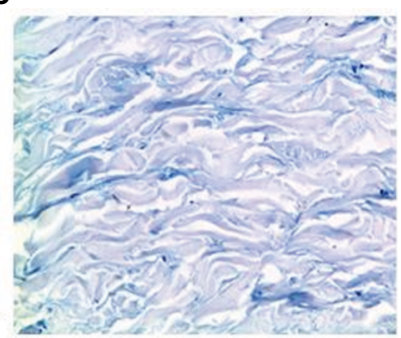

Figure 7 Alcian blue-stained dermal tissue from ex vivo hydrocortisone study.

Notes: Proteoglycans are stained in deep blue (magnification 200x). (A) Untreated control tissue; (B) hydrocortisone control tissue; and (C) hydrocortisone- and serumtreated tissue.

Table 5 Clinical studies subject characteristics

\begin{tabular}{llll}
\hline Study & $\begin{array}{l}\text { Number of } \\
\text { subjects }\end{array}$ & $\begin{array}{l}\text { Women, } \\
\text { n (\%) }\end{array}$ & $\begin{array}{l}\text { Age, years } \\
\text { (min-max) }\end{array}$ \\
\hline $\begin{array}{l}\text { Clinical study I: hydration } \\
\text { kinetics }\end{array}$ & 20 & $20(100)$ & $49.2(21-67)$ \\
$\begin{array}{l}\text { Clinical study 2: efficacy } \\
\text { and tolerability under }\end{array}$ & 30 & $30(100)$ & $37(21-45)$ \\
$\begin{array}{l}\text { dermatological control } \\
\begin{array}{l}\text { Clinical study 3: consumer } \\
\text { study }\end{array}\end{array}$ & 105 & $105(100)$ & $35.7(18-45)$ \\
\hline
\end{tabular}

(5.08) at $6 \mathrm{~h}$, representing a 39.3, 12.6, and $7.2 \%$ increase, respectively $(P<0.01$ for all) (Figure 8$)$. Skin moisture on Skicon reading (microsiemens $[\mu \mathrm{S}])$ also increased significantly at 1,3 , and $6 \mathrm{~h}$ by $234.4,78.2$, and $44.6 \%$, respectively $(P<0.001$ for all) (Figure 9).

\section{Efficacy and acceptability under dermatological control}

The results of the efficacy questionnaire are shown in Table 6. Of note, $83 \%$ of participants found that the product provided immediate hydration, $83 \%$ of participants found that the skin appeared brighter, and $77 \%$ of participants found that wrinkles, if present, had decreased or softened. The cosmetic qualities were evaluated favorably, and $80 \%$ of participants liked the product overall. No abnormal clinical signs were observed by

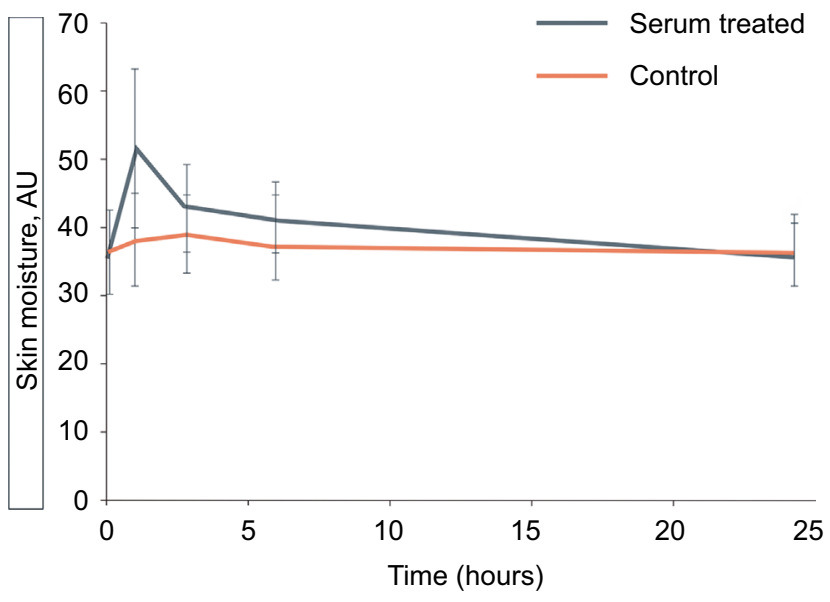

Figure 8 Hydration kinetics study of skin moisture levels over time as measured with corneometer.

Note: Serum treated vs control.

the dermatologist in any patient after 4 weeks of serum use. Good acceptability was reported by 25 of the 30 subjects. In four of the other five subjects, subject-reported reactions included redness, itching, tightness, dryness, and tears, though these were not observed by the dermatologist. One subject reported itching and comedones and requested to leave the study. Upon the dermatologist's count of retentional and inflammatory elements (comedogenic potential), no statistically significant difference was observed between days 1 and 29. 


\section{Consumer test}

The investigational product was liked overall by $95 \%$ of the 105 women involved (mean age 35.7 years). Results of the efficacy questionnaire are shown in Table 6. A total of $94 \%$ of participants felt that their skin was immediately moisturized, $87 \%$ of participants noted an immediate antifatigue effect, and $78 \%$ of participants reported that wrinkles, if present, had decreased or softened.

Two subjects reported cutaneous manifestations. One subject with oily skin and no previous history of reactions reported comedones on the face, and another subject with mixed oily skin and a history of reactions reported comedones and itching on the cheeks and chin. However, $77 \%$ of the participants found that marks or blemishes, if present, had decreased. No ocular manifestations were reported.

When asked to rate the product overall, $89 \%$ of participants scored the product 7 out of 10 .

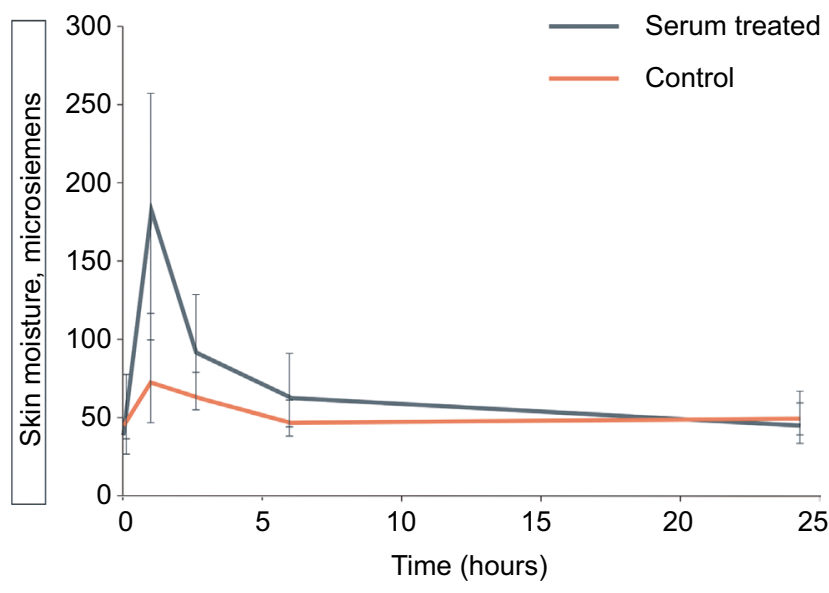

Figure 9 Hydration kinetics study of skin moisture levels over time as measured with Skicon hygrometer.

Note: Serum treated vs control.

\section{Discussion}

These studies have largely demonstrated that the application of this serum resulted in a preserved dermal matrix structure and effective antioxidant protection when skin was exposed to photodamage and simulated chronological aging. The findings from the ex vivo studies were further supported by clinical findings of immediate and sustained hydration measurements up to $6 \mathrm{~h}$ and self-perceived immediate moisturizing and antifatigue effects, brighter skin, wrinkle reduction, and desire to continue using the product.

Several previous studies ${ }^{10-18}$ have demonstrated the actions of the individual ingredients included in the formulation objective of current investigation. Results from our study provide further evidence on this synergistic combination of ingredients.

L-Ascorbic acid, the active form of vitamin C, is a potent antioxidant. It donates electrons, protects against ROS, ${ }^{10}$ stabilizes collagen crosslinking, ${ }^{11}$ increases collagen production by fibroblasts, ${ }^{12}$ inactivates free radicals induced by ultraviolet rays, and regenerates vitamin $\mathrm{E},{ }^{10}$ another potent antioxidant. However, it is inherently unstable. ${ }^{13}$ To preserve its antioxidant properties, the L-ascorbic acid in this serum was stabilized by adding ergothioneine and tocopherol and the product was packaged under conditions designed to limit oxidation - single-use packaging to avoid excess exposure to air, amber glass ampoules (an established strategy to prevent light penetration), ${ }^{12}$ and under an inert atmosphere of argon and nitrogen. One of the potential side effects of high concentrations of vitamin $\mathrm{C}$ is that it has the potential to cause skin irritation, or when oxidized, leave a yellowish tinge on the skin. ${ }^{13}$ In the clinical study, no reactions were observed by the dermatologist, although a few patients did report some form of reaction (comedones or itching). We cannot rule out that this

Table 6 Results from subjective efficacy questionnaire under dermatological control and consumer test questionnaire

\begin{tabular}{lll}
\hline Questionnaire item & \% of subjects in agreement & Consumer test \\
\cline { 2 - 3 } & $\begin{array}{l}\text { Efficacy and tolerability under } \\
\text { dermatological control }\end{array}$ & 94 \\
\hline The product provided immediate hydration & 83 & 87 \\
The product had an immediate antifatigue effect (flash effect) & 70 & 91 \\
Skin feels more hydrated day by day & 87 & 78 \\
Wrinkles, if present, decreased or softened & 77 & 78 \\
Lines, if present, decreased or softened & 70 & 95 \\
The skin of the face looks brighter & 83 & 90 \\
Skin feels more rested & 87 & 91 \\
Skin feels healthier & 87 & 95 \\
Liked the product overall & 80 & 89 \\
Would like to continue using the product & 55 & 82 \\
Would buy the product independently of the price & 60 & 95 \\
Overall you liked this product & 80 & \\
\hline
\end{tabular}


may have been caused by the product, and longer tolerability studies would be useful; noncomedogenicity was established.

Ergothioneine is a thiol that forms part of the skin's antioxidant system. ${ }^{14}$ Its effect on fibroblast activity has been reported in an earlier study as has its ability to suppress UVB-induced expression of MMP-1. ${ }^{15}$ Additionally, ergothioneine has been reported to help maintain L-ascorbic acid in its reduced state ${ }^{16}$ and a synergistic relationship between ergothioneine and vitamin $\mathrm{C}$ has been described. ${ }^{17}$

The combination of proteoglycan-stimulating peptide with fragmented proteoglycans (Ultraglycans ${ }^{\mathrm{TM}}$ ) was included in the product with the aim of stimulating fibroblast activity and thus maintaining ECM structural integrity. Earlier published reports suggest that proteoglycan synthesis can be stimulated by the application of certain topical peptides (such as copper peptide and carnosine) ${ }^{18}$ but to our knowledge, the effects of the particular peptide in the tested product (tetradecyl aminobutyroylvalylaminobutyric urea trifluoroacetate) have not been published. Barba et $\mathrm{al}^{19}$ have reported the effects of a serum containing fragmented proteoglycans from soybeans on collagen and proteoglycan levels in vitro, although their results are not directly comparable to ours due to differences in methodology. Our study was a comparison of aged and serum-treated skin against aged untreated skin and nonaged skin, and we found that levels in serum-treated samples were close to those of healthy, nonaged skin. Although the levels in our study did not increase beyond the levels found in healthy skin, the study duration was shorter, at 10 days rather than 30 days, and the skin, as mentioned, had been damaged. The matrix changes observed in the short study time frame was one of our most salient findings.

Finally, hyaluronic acid is known to bind large amounts of water in the skin, improving skin turgor; ${ }^{20}$ low molecular weight hyaluronic acid is able to pass to the dermis and stimulate fibroblast activity; ${ }^{21,22}$ our clinical results measured with corneometer and Skicon demonstrate the effects of this action at a clinical level.

The main strength of these studies is the combination of ex vivo skin explant studies with clinical studies in volunteers, showing objectively how the changes in markers of oxidation and ECM degradation occurred and how such effects were perceived by participants. The ex vivo studies used human skin explants, which have been described as "similar to in vivo clinical human skin tests" 23 and provide useful mechanistic information on changes in skin. Skin hydration was assessed on the forearms, considered representative of facial skin, ${ }^{24}$ and used the widely adopted, reproducible, objective methods of corneometer and Ski- con hygrometer. ${ }^{25}$ The limitations of these studies include the subjective nature of some of the clinical questionnaire items, as descriptions can be interpreted differently by different individuals; a placebo group and larger sample size over a longer study duration would have made the results more robust. The design could have been improved further by adding some objective evaluations such as photographic analysis, or wrinkle count and depth.

\section{Conclusion}

These studies demonstrate that this serum containing L-ascorbic acid, ergothioneine, hyaluronic acid, a proteoglycan-stimulating peptide, and fragmented proteoglycans was effective in protecting against solar-induced oxidative damage and simulated chronological aging, maintaining optimum levels of dermal matrix components including structural proteins, proteoglycans, and moisture, which resulted in more hydrated skin. Participants perceived that the product improved the skin's appearance in terms of brightness, wrinkle reduction, and hydration.

\section{Acknowledgment}

Medical writing assistance was provided by $\mathrm{J}$ Marshall.

\section{Disclosure}

AG, MN, PV-M, and CG are employees of ISDIN SA, who sponsored these studies. JP is an external advisor to ISDIN SA. The authors report no other conflicts of interest in this work.

\section{References}

1. Fore J. A review of skin and the effects of aging on skin structure and function. Ostomy Wound Manage. 2006;52(9):24-35.

2. Kaisers W, Boukamp P, Stark H, et al. Age, gender and UV-exposition related effects on gene expression in in vivo aged short term cultivated human dermal fibroblasts. PLoS One. 2017;12(5):e0175657.

3. Poljšak B, Dahmane R, Godić A. Intrinsic skin aging: the role of oxidative stress. Acta Dermatovenerol Alp Pannonica Adriat. 2012;21(2):33-36.

4. Karran P, Brem R. Protein oxidation, UVA and human DNA repair. DNA Repair (Amst). 2016;44:178-185.

5. legifrance.gouv.fr. Law no. 2011-814 of 7 July on bioethics (article 18). JORF No. 0157 of 8 July 2011 page 11826 text no. 1 NOR: ETSX1117652L. Available from: https://www.legifrance.gouv.fr/eli/ loi/2011/7/7/ETSX1117652L/jo/texte. Accessed February 27, 2018. French.

6. Autio P, Oikarinen A, Melkko J, Risteli J, Risteli L. Systemic glucocorticoids decrease the synthesis of type I and type III collagen in human skin in vivo, whereas isotretinoin treatment has little effect. $\mathrm{Br}$ J Dermatol. 1994;131(5):660-663.

7. Rasmussen E, Goldberg A. Use of fluorescent redox indicators to evaluate cell proliferation and viability. In Vitro and Molecular Toxicology. AM Goldberg, editor New York, NY, USA: Mary Ann Liebert; 1999:47-58.

8. Osborne R, Perkins M. An approach for development of alternative test methods based on mechanisms of skin irritation. Food Chem Toxicol. 1994;32(2):133-142. 
9. World Medical Association [webpage on the Internet]. Declaration of Helsinki - Ethical Principles for Medical Research Involving Human Subjects; 2013. Available from: https:/www.wma.net/policies-post/ wma-declaration-of-helsinki-ethical-principles-for-medical-researchinvolving-human-subjects/. Accessed February 27, 2018.

10. Chaudière J, Ferrari-Iliou R. Intracellular antioxidants: from chemical to biochemical mechanisms. Food Chem Toxicol. 1999;37(9-10): 949-962.

11. Grinnell F, Fukamizu H, Pawelek P, Nakagawa S. Collagen processing, crosslinking, and fibril bundle assembly in matrix produced by fibroblasts in long-term cultures supplemented with ascorbic acid. Exp Cell Res. 1989;181(2):483-491.

12. Boyera N, Galey I, Bernard B. Effect of vitamin C and its derivatives on collagen synthesis and cross-linking by normal human fibroblasts. Int J Cosmet Sci. 1998;20(3):151-158.

13. Telang P. Vitamin C in dermatology. Indian Dermatol Online J. 2013;4(2): 143-146.

14. Dong K, Damaghi N, Kibitel J, Canning M, Smiles K, Yarosh D. A comparison of the relative antioxidant potency of L-ergothioneine and idebenone. J Cosmet Dermatol. 2007;6(3):183-188.

15. Obayashi K, Kurihara K, Okano Y, Masaki H, Yarosh DB. L-Ergothioneine scavenges superoxide and singlet oxygen and suppresses TNF-alpha and MMP-1 expression in UV-irradiated human dermal fibroblasts. J Cosmet Sci. 2005;56(1):17-27.

16. Coassin M, Tomasi A, Vannini V, Ursini F. Enzymatic recycling of oxidized ascorbate in pig heart: one-electron vs two-electron pathway. Arch Biochem Biophys. 1991;290(2):458-462.
17. Asmus K, Bensasson R, Bernier J, Houssin R, Land EJ. One-electron oxidation of ergothioneine and analogues investigated by pulse radiolysis: redox reaction involving ergothioneine and vitamin C. Biochem J. 1996;315(pt 2):625-629.

18. Shagen $\mathrm{S}$. Topical peptide treatments with effective anti-aging results. Cosmetics. 2017;4(2):16.

19. Barba C, Alonso C, Sánchez I, Suñer E, Sáez-Martín L, Coderch L. Soybean-fragmented proteoglycans against skin aging. J Cosmet Laser Ther. 2017;19(4):237-244.

20. Ghersetich I, Lotti T, Campanile G, Grappone C, Dini G. Hyaluronic acid in cutaneous intrinsic aging. Int J Dermatol. 1994;33(2):119-122.

21. Cilurzo F, Vistoli G, Gennari C, et al. The role of the conformational profile of polysaccharides on skin penetration: the case of hyaluronan and its sulfates. Chem Biodivers. 2014;11(4):551-561.

22. Nashchekina Y, Raydan M. Noninvasive penetration of $5 \mathrm{~nm}$ hyaluronic acid molecules across the epidermal barrier (in vitro) and its interaction with human skin cells. Skin Res Technol. 2018;24(1):129-134.

23. Frade M, Andrade T, Aguiar A, et al. Prolonged viability of human organotypic skin explant in culture method (hOSEC). An Bras Dermatol. 2015;90(3):347-350.

24. Bazin R, Fanchon C. Equivalence of face and volar forearm for the testing of moisturizing and firming effect of cosmetics in hydration and biomechanical studies. Int J Cosmet Sci. 2006;28(6):453-460.

25. Clarys P, Clijsen R, Taeymans J, Barel A. Hydration measurements of the stratum corneum: comparison between the capacitance method (digital version of the Corneometer CM 825®) and the impedance method (Skicon-200EX®). Skin Res Technol. 2012;18(3):316-323.
Clinical, Cosmetic and Investigational Dermatology

\section{Publish your work in this journal}

Clinical, Cosmetic and Investigational Dermatology is an international, peer-reviewed, open access, online journal that focuses on the latest clinical and experimental research in all aspects of skin disease and cosmetic interventions. This journal is included on PubMed. The manuscript management system is completely online

\section{Dovepress}

and includes a very quick and fair peer-review system, which is all easy to use. Visit http://www.dovepress.com/testimonials.php to read real quotes from published authors 